\title{
The inhibitory effect of Gualou Guizhi Decoction on post-ischemic neuroinflammation via miR-155 in MCAO rats
}

\author{
Jinbo Yang ${ }^{1,2}$, Xiaoqin Zhu ${ }^{1,2}$, Haixia Hu ${ }^{1,2}$, Xinjun Lin $^{3}$ \\ ${ }^{1}$ Academy of Integrative Medicine, Fujian University of Traditional Chinese Medicine, Fuzhou, China; ${ }^{2}$ Fujian Key Laboratory of Integrative \\ Medicine on Geriatrics, Fuzhou, China; ${ }^{3}$ Institute of Integrated Chinese and Western Medicine, Fujian University of Traditional Chinese Medicine, \\ Fuzhou, China \\ Contributions: (I) Conception and design: H Hu; (II) Administrative support: X Zhu; (III) Provision of study materials or patients: X Lin; (IV) \\ Collection and assembly of data: J Yang; (V) Data analysis and interpretation: J Yang; (VI) Manuscript writing: All authors; (VII) Final approval of \\ manuscript: All authors. \\ Correspondence to: Haixia Hu. Academy of Integrative Medicine, Huatuo Road, Minhou Shangjie, Fuzhou, China. Email: asunnyhaixia@163.com.
}

\begin{abstract}
Background: Gualou Guizhi Decoction (GLGZD) is commonly used to treat stroke. The present study investigated the potential roles of GLGZD on inflammation involving microRNA-155 (miR-155) in a model of ischemic stroke using middle cerebral artery occlusion (MCAO) rats.

Mothods: Sprague-Dawley rats were randomly divided into three groups: Sham operated group, MCAO model group, and GLGZD treatment group. The ischemic model was established by $2 \mathrm{~h}$ left MCAO followed by reperfusion. Neurological deficits were evaluated with a modified Ashworth scale in each group. The changes in individual paw parameters were assessed by Catwalk gait analysis. Inflammatory cytokines were measured by enzyme linked immunosorbent assay (ELISA) and protein levels and gene expression related to inflammation were detected by Western blot and quantitative reverse transcription-PCR (qRTPCR) assays, respectively. The expression of inflammatory signaling proteins was additionally detected by immunohistochemistry.
\end{abstract}

Results: Treatment of MCAO rats with GLGZD improved neuronal defects and limb motivity. Additionally, GLGZD was able to inhibit miR-155 upregulation, resulting in down-regulation of miR155-targeted molecules in MCAO rats, including suppressor of cytokine signaling 1 (SOCS1), inhibitor of nuclear factor kappa-B kinase (IKK), mothers against decapentaplegic homolog 2 (SMAD2) and CCAAT/ enhancer binding protein beta (СЕВР $\beta)$. Meanwhile, the production of anti-inflammatory cytokines was dramatically enhanced by GLGZD treatment when comparing with the MCAO model group.

Conclusions: In conclusion, GLGZD down-regulates miR-155, mediating subsequent neuroinflammation and resulting in neuroprotection which contributes to reduced spasticity after ischemic stroke.

Keywords: Gualou Guizhi Decoction (GLGZD); neuroinflammation; miR-155; inflammation; stroke

Submitted Mar 02, 2020. Accepted for publication Sep 10, 2020.

doi: 10.21037/apm-20-518

View this article at: http://dx.doi.org/10.21037/apm-20-518

\section{Introduction}

Stroke is a common and multiple disease with high morbidity, mortality and disability rate which work together to seriously endanger human health (1). Spasticity is common challenging condition of stroke, stemming from neuronal injury and resulting in limited limb mobility
$(2,3)$. Although the pathogenesis of stroke involves various mechanisms such as apoptosis, inflammation, oxidative stress and excitotoxicity, mounting evidence shows that neuroinflammation plays a key role in the pathogenetic mechanisms of acute ischemic stroke. In past decades, researchers have held the view that inflammation was harmful to neuronal protection during 
brain ischemia/reperfusion. When cerebral ischemia and hypoxia occur, microglia and astrocytes are activated, secreting inflammatory factors and mediators such as tumor necrosis factor-a (TNF- $\alpha$ ), the interleukins (IL) and transforming growth factor-beta (TGF- $\beta$ ), all which activate inflammatory cells. Inflammatory cells under these conditions result in neuronal cell death and aggravation of brain injury (4-6). Recent reports have identified that several critical inflammation-related miRNAs, capable of regulating gene/protein expression by inhibiting mRNA translation $(7,8)$. Recent findings have also verified that miR-155 is highly expressed in brain tissue and involved in post-stroke neuroinflammation by targeting specific molecules $(9,10)$.

Gualou Guizhi Decoction (GLGZD) was in the 'Essentials from the Golden Cabinet' records, a classic clinical book of Traditional Chinese Medicine. GLGZD is composed of six herbal ingredients: Trichosanthes kirilowii Maxim., Paeonia lactiflora Pall., Cinnamomum cassia Presl., Glycyrrbiza uralensis Fisch., Zingiber officinale Rosc. and Ziziphus jujuba Mill. Recent studies suggest that GLGZD can reduce the activation of microglia, reducing nerve injury during ischemia/reperfusion and playing a role in nerve protection (11-14).

Considering the neuroprotective role of GLGZD in inflammation conditions along with their role in ischemia reperfusion injury, the present study was designed to further investigate the effect of GLGZD on miR-155 and various inflammatory mediators in MCAO rats. MCAO rat was proved to be a reliable and effective model in experimental stroke studies for yielding consistently larger infarcts and greatly produced neuron-inflammatory mediators. The results of this study reveal fundamental evidence suggesting a prospective therapeutic target for ischemic stroke. Our study also revealed targets of GLGZD capable of modulating the inflammatory response following ischemic challenge. We present the following article in accordance with the ARRIVE reporting checklist (available at http:// dx.doi.org/10.21037/apm-20-518).

\section{Methods}

\section{Reagents}

Real time-quantitative polymerase chain reaction (RTqPCR) reagents were supplied by Takara Bio, Inc. (Otsu, Japan). The IL10 and TGF- $\beta$ were analyzed from using enzyme linked immunosorbent assay (ELISA) kits from
R\&D Systems, Inc. (Minneapolis, MN, USA). Primary antibodies against iNOS (cat. no. sc-7271), Cox-2 (cat. no. sc-166475) and $\beta$-actin (cat. no. sc-47778) were purchased from Santa Cruz Biotechnology, Inc (Dallas, TX, USA). Western blotting reagents were supplied by Beyotime Biotechnology, Inc. (Shanghai, China). The monoclonal antibodies targeting SOCS1 (cat. no. ab62584), IKK (cat. no. ab178870), SMAD2 (cat. no. ab40855) and CEBP $\beta$ (cat. no. ab32358), and rabbit secondary horseradish peroxidase (HRP)-conjugated immunoglobulin G antibody (cat. no. ab6747) were obtained from Abcam Biotechnology, Inc. (Cambridge, MA, USA).

\section{Animals}

Male Sprague-Dawley rats (age, 6 weeks; weight, 200-250 g) which were obtained from Shanghai SLAC Laboratory Animal Co., Ltd. (Shanghai, China) were employed for experiments. Animals were made to adapt the standardized laboratory facilities for seven days and housed in a room on a 12-hour light/dark cycle with free access to food and pure water. Experimental procedures and the care of animals were performed strictly in conformity with international ethical guidelines requirement and the National Institutes of Health Guide concerning the Care and Use of Laboratory Animals. All experimental protocols were approved by the Institutional Animal Care and Use Committee in Fujian University of Traditional Chinese Medicine (Fuzhou, China) (No.: 20190016).

\section{Preparation of herbal extracts}

Medicinal plants were obtained from Guo Yi Tang Chinese Herbal Medicine Store (Fujian, China). The preparation of GLGZD included six plants: Trichosanthis radix, Ramulus cinnamomi, Paeonia lactiflora, Glycyrrbiza radix, Zingiber officinale Roscoe and Fructus jujubae at a ratio of $3: 3: 3: 2: 3: 3$. After incubating in double distilled water for $30 \mathrm{~min}$, the mixture was then heated to $100{ }^{\circ} \mathrm{C}$ followed by refluxing twice in $2 \mathrm{~h}$. Subsequently, the collected extract was evaluated at a final concentration of $1.16 \mathrm{~g} / \mathrm{mL}$ after continuous filtration and concentration (15).

\section{Establishment of animal model and experimental grouping}

$10 \%$ chloral hydrate $(350 \mathrm{mg} / \mathrm{kg}$, intraperitoneal injection, Sinopharm Chemical Reagent Co., Ltd., Shanghai, China) 
was used for mice anesthesia, subsequently the middle cerebral artery was occluded for $2 \mathrm{~h}$ with sutures. Briefly, monofilament nylon sutures coated with poly-L-lysine (Beijing Sunbio Biotech Co., Ltd., Beijing, China) were inserted into the internal carotid artery (ICA) $(\sim 8-20 \mathrm{~mm}$ beyond the carotid artery bifurcation) inhibiting blood flow. After $2 \mathrm{~h}$, the suture was withdrawn slowly by $\sim 10 \mathrm{~mm}$ to restore blood reperfusion. During all procedures, body temperature of rats was maintained at physiological body temperature to prevent hypothermia. Rats were randomized into three groups ( $\mathrm{n}=15$ per group): Sham group in which rats received the same surgery without cerebral artery occlusion; the MCAO model group in which rats underwent ischemia surgery with no GLGZD treatment. Finally, in the GLGZD group, rats were subjected to MCAO surgery and intragastrically administrated with GLGZD $(1.16 \mathrm{~g} / \mathrm{mL})$ for seven days. Rats were randomly assigned to the following groups after MCAO: (I) neurological function ( $\mathrm{n}=10$ ); (II) catwalk analysis $(\mathrm{n}=6)$; (III) anti-inflammatory cytokines assessment ( $\mathrm{n}=3)$; (IV) western blotting assay $(\mathrm{n}=3)$; (V) immunohistochemistry staining $(\mathrm{n}=5)$. All experiments were repeated at least 3 times.

\section{Assessment of neural and functional defects}

Neurological function was assessed with the Zea Longa 5-point scoring method (16). The evaluations of neurological deficits were performed for every group at $2 \mathrm{~h}$ and 7 days after reperfusion in a blinded manner. Briefly, 0 , normal movement without any neurological impairment; 1 , the right forepaw is complete unable to move; 2, circling inwards when walking; 3 , complete failure to the right side; and 4 , complete loss of spontaneous motor activity.

\section{Catwalk gait analysis}

Cortical function was automatically analyzed by the CatWalk system (Noldus Information Technology, The Netherlands), which can be used to assess spontaneous movement of rats in an accurate and objective manner. After undergoing CatWalk pre-training, the rat ran across a glass walkway and gait parameters were recorded by a highspeed digital camera and transformed into digital images. The rats which stopped and were not able to pass the glass plate smoothly were excluded from the experimental group. The changes in paw-related parameters of all groups were automatically measured by the CatWalk system, including print area $\left(\mathrm{cm}^{2}\right)$ representing the maximum area of the print of a paw, stride length $(\mathrm{cm})$ indicating the distance between successive placements of the same paw, and swing speed $(\mathrm{cm} / \mathrm{s})$ indicating the speed (distance unit/second) of the paw during a swing.

\section{Measurement of inflammatory cytokine by ELISA}

The levels of TGF- $\beta$ and IL-10 were quantified by ELISA kits (R\&D Systems, Inc., Minneapolis, MN, USA) following the protocols provided by the manufacturer. An automatic biochemical analyzer (BioTek 8008, Bad Friedrichshall, Germany) was used to measure protein concentrations.

\section{Reverse transcription-quantitative polymerase chain reaction ( $R T-q P C R)$}

Tissues from the ischemic hemisphere was isolated from the brain of all groups after experimental treatment. Trizol reagent (Invitrogen, Carlsbad, CA, USA) was used to isolate total RNA according to manufacturer's instructions. RNA was then converted into cDNA and amplified with $\mathrm{SYBR}^{\circledR}$ Premix Ex TaqTM (DRR420A, TaKaRa Bio Inc.) with Applied Biosystems Prism 7500 (Applied Biosystems Life Technologies, Carlsbad, CA, USA). The primers for RTPCR were the following: miR-155: $\mathrm{F}$ (forward primer): 5'-TATCAACACCACCTGCGACC-3'; R(reverse primer): 5'-ACTCGGTCAGAAAGCCAGTG-3'; SOCS1: F: 5'-GGAGT CTGTGCTCTGGGATT-3', R: 5'-T GAGGGAGTGAAGGAGCAAC-3'; SMAD2: F: 5'-GAGTACCTGAACCGGCATCT-3'; R: 5'-GGTA TGCA CCCAGAGTGATG-3'; IKK: F: 5'-GCCC TTAGAACTGGAGGGAGG-3'; R: 5 '-CCAGGC CCCTATCTGTAGCA-3'; CEBPß: F: 5'-AGCT TGGAACGGTACGCTAA - 3 '; R: 5 ' -GAGTC CACTGACTTGCTCCC-3'; $\beta$-actin served as an internal gene: F: 5'-ACTCTGTGTGGATTGGTGGC-3', R: 5'-AGAAAGGGTGTAAAACGCAGC-3'; IL10: F: 5'-TGGGTAGACAGCAGTGCCAC-3', R: 5'-GCCCACAAGATGGACAGGG-3'; TGF $\beta$ : F: 5 '-GGCACCATCCATGACATGAACCG-3'; R: 5'-GCCGTACACAGCACTTCTTCTCTG-3'. Reaction conditions of amplification were designed according to the kit instructions. The relative gene of interest was normalized to the internal control $\beta$-actin and results were analyzed using the $2-\Delta \Delta \mathrm{Ct}$ method. 


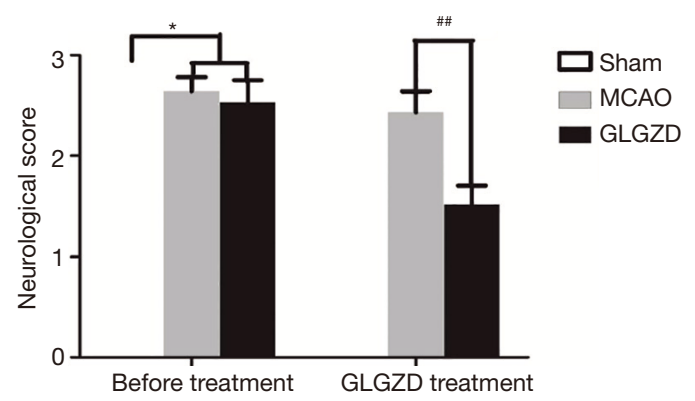

Figure 1 GLGZD have positive influence on the neuronal deficits in MCAO rats. Neurological behavior score was assessed by Zea Longa method scoring from 0-4 point. Data are presented as the mean \pm standard error of the mean $(\mathrm{n}=10) .{ }^{*} \mathrm{P}<0.05$ vs. the sham group; ${ }^{\# \#} \mathrm{P}<0.01$ vs. the MCAO group. GLGZD, Gualou Guizhi Decoction; MCAO, middle cerebral artery occlusion.

\section{Determination of protein expression by Western blot analysis}

The total proteins of the ipsilateral cortex were extracted using lysis buffer (containing a protease inhibitor). Protein concentration was determined by a BCA Protein Assay Kit (Thermo Fisher Scientific). Equal amounts of proteins were denatured in $100^{\circ} \mathrm{C}$ boiling water for $3 \mathrm{~min}$ and separated by sodium dodecyl sulfate polyacrylamide gel electrophoresis (SDS-PAGE), and then transferred to a PVDF membrane (Millipore, Billerica, MA, USA). The membrane was blocked with $10 \%$ dried milk solution for $1 \mathrm{~h}$ and incubated with iNOS and Cox-2 primary antibodies $(1: 1,000)$ at $4{ }^{\circ} \mathrm{C}$ overnight. After washing three times in TBST, the membranes were exposed to the second antibody $(1: 5,000)$ at room temperature for $1 \mathrm{~h}$, then washed by TBST three times. Finally, protein immunoblots were detected using ECL detection reagent (P0018, Beyotime Biotechnology) and ChemiDoc instrument (Bio-Rad). $\beta$-actin mRNA was treated as the internal control. Image analysis of the gel bands densitometry was carried out with Bio-Image Analysis System (Bio-Rad, Hercules, CA, USA).

\section{Immunobistochemistry}

Following treatment, rats were perfused with $0.01 \mathrm{~mol} / \mathrm{L}$ PBS and followed by ice-cold 4\% paraformaldehyde through the intracardiac route. Brain were dissected, fixed in $4 \%$ paraformaldehyde, embedded in paraffin and cut into $5 \mu \mathrm{m}$ coronary sections. After washing with PBS for $15 \mathrm{~min}$, the brain sections were blocked with normal horse serum. Tissue sections were then incubated with anti-SOCS1, anti-SMAD2, anti-IKK and anti-CEBP (Beijing Biosynthesis Biotechnology Co., Ltd., Beijing, China) overnight at $4^{\circ} \mathrm{C}$. After being washed in PBS, sections were incubated for $1 \mathrm{~h}$ at RT with appropriate secondary antibody. Immunopositive antigens were subsequently detected using a DAB substrate System (Maixin Biotechnology, Fuzhou, China). Tissue sections were then observed under light microscope (400x) and representative photographs were taken and analyzed by Image J software (Imaging Research, St. Catharines, ON, Canada).

\section{Statistical analysis}

All animals were randomly assigned to treatment conditions. Results are shown as the mean \pm SD. error. Data from each of the three groups were analyzed by performing one-way analysis of variance (ANOVA) with post hoc Banferroni tests using SPSS version 16.0 (SPSS, Inc., Chicago, IL, USA). $\mathrm{P}$ value $<0.05$ were indicated as statistical significance.

\section{Results}

\section{GLGZD treatment improves functional outcome after ischemia in MCAO rats}

The animals in each experimental group were in good condition and met the weight requirements before the experiment. Stroke injury modeling induced significant neuronal and functional deficits. As shown in Figure 1, the neurological score was dramatically increased in the MCAO group when comparing with the sham group, $(\mathrm{P}<0.05)$. The GLGZD treatment group exhibited significantly improved neurological functional outcomes after 7 days of treatment when comparing with the MCAO group $(\mathrm{P}<0.01)$. Similarly, in catwalk gait analysis, there were significant differences in multiple gait Parameters, reflecting movement across the different experimental groups. As shown in Figure 2, owing to the ischemic limb spasm, the print areas of the right limbs, the stride length of all limbs and swing speed in the MCAO group demonstrated significant decreases $(\mathrm{P}<0.05)$ compared with the sham group. In addition, GLGZD effectively improved the recovery of all parameters which were reduced by MCAO $(\mathrm{P}<0.05)$.

\section{GLGZD up-regulates the expression of anti-inflammatory mediators in the MCAO rats}

Under normal physiological conditions, anti-inflammatory 

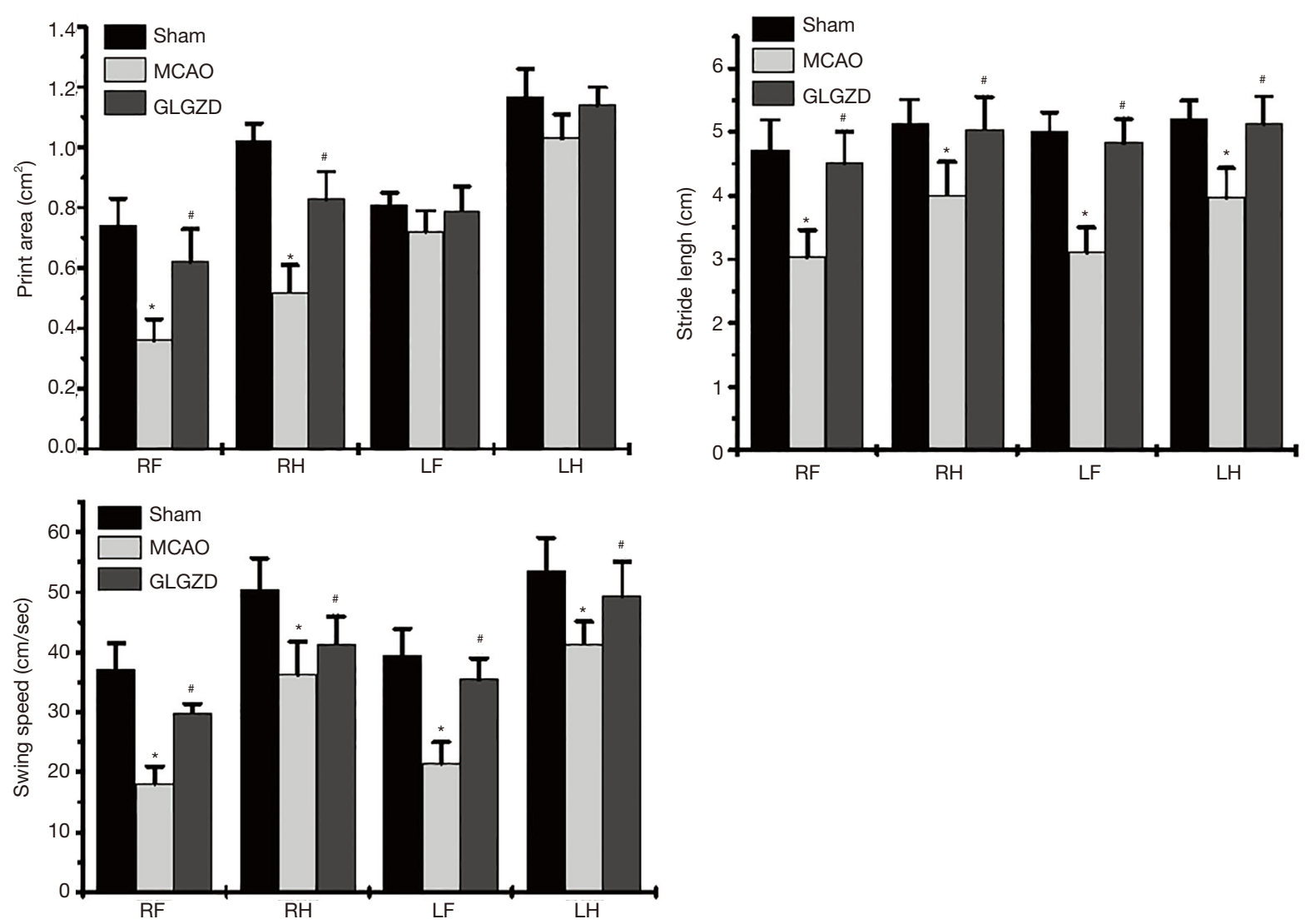

Figure 2 GLGZD affected the gait parameters detected by Catwalk analysis in MCAO rats. RF, right front paw; RH, right hint paw; LF, left front paw; LH, left hint paw. Print area, stride length and swing speed represented the moving activity of rats in different experimental groups ( $\mathrm{n}=6$, mean $\pm \mathrm{SEM},{ }^{*} \mathrm{P}<0.05$ compared to sham group, ${ }^{\#} \mathrm{P}<0.05$ compared to MCAO group). GLGZD, Gualou Guizhi Decoction; $\mathrm{MCAO}$, middle cerebral artery occlusion.

cytokines are expressed at low levels, however, they are dramatically increased under inflammation states, particularly following ischemic stroke. In this study, there was no obvious change in the release of IL10 or TGF $\beta$ in the peripheral blood of MCAO group rats comparing with the sham group. Conversely, GLGZD treatment induced a significant increase in protein expression of IL10 and TGF $\beta$ compared with the MCAO model group $(\mathrm{P}<0.05)$. Simultaneously, the gene expression levels demonstrated similar changes in these three groups $(\mathrm{P}<0.05$, Figure 3$)$.

\section{GLGZD ameliorates the expression of inflammatory mediators in MCAO rats}

To investigate the inflammatory mediators associated with neuronal damage in the ischemic ipsilateral hemisphere, indicators of oxidative damage were evaluated. The results
(Figure 4) indicated that MCAO rats exhibited a marked increase $(\mathrm{P}<0.05)$ in the translational levels of iNOS and Cox-2 compared to the sham operation group. On the other hand, GLGZD remarkably reduced iNOS and Cox-2 expression when compared with the MCAO group $(\mathrm{P}<0.05$ for iNOS, $\mathrm{P}<0.01$ for Cox-2, $\mathrm{n}=3$ ).

\section{miR155-mediated signaling is involved in the GLGZD treatment of $\mathrm{MCAO}$ rats}

To explore the underlying mechanism by which GLGZD regulates neuroinflammation, miR-155 and its target genes were measured using Western blot or RT-PCR assay. As shown in Figure 5, miR-155 levels were increased and its associated targets, SOCS1, IKK, SMAD2 and CEBP $\beta$, were decreased in MCAO rats compared with those of the sham group (Figure $5 A, \mathrm{P}<0.05$ for SOCS1, IKK and CEBP $\beta$, 

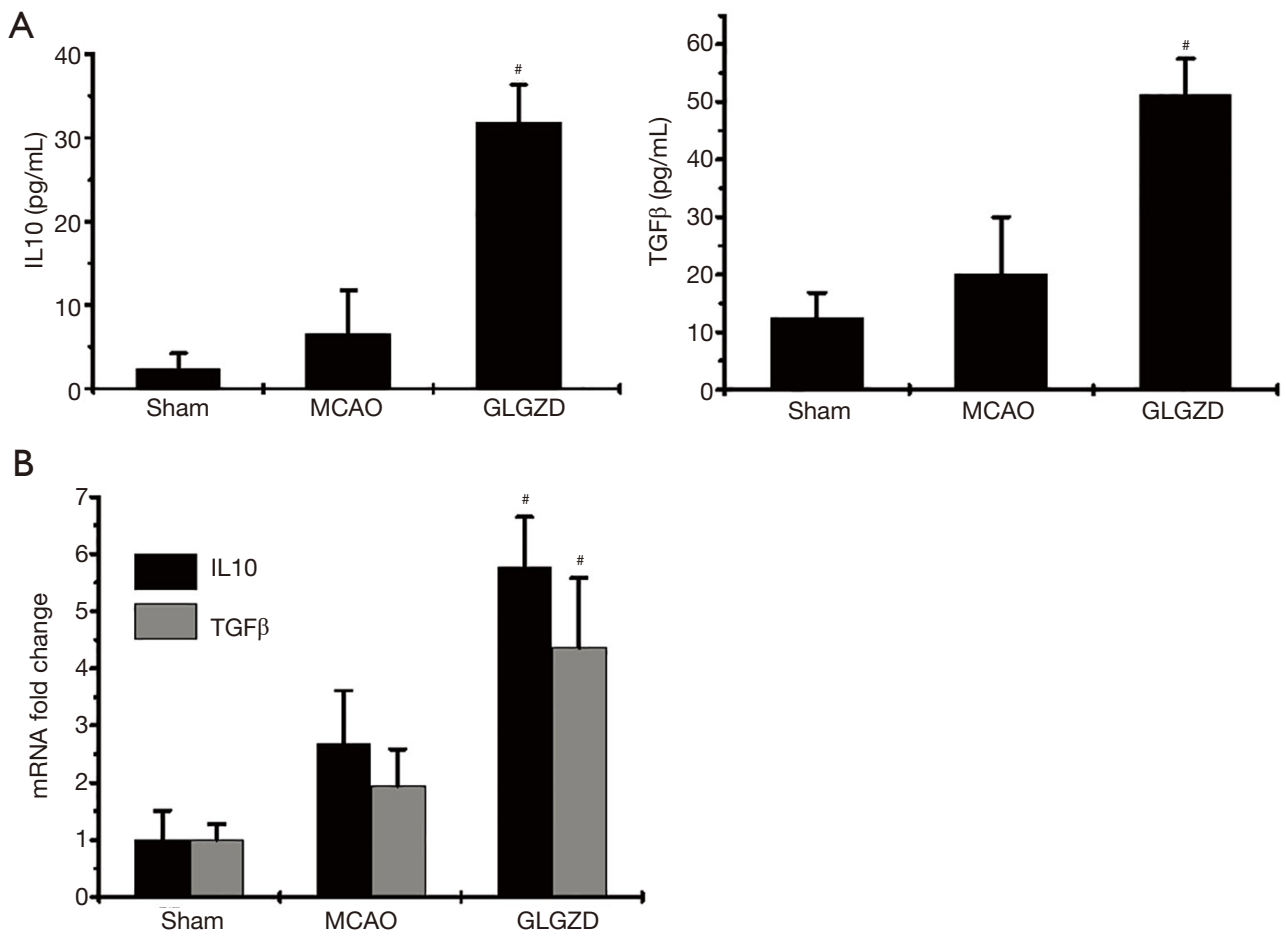

Figure 3 The effect of GLGZD on the protein and gene expression of anti-inflammatory cytokines in MCAO rats. (A) The production of interleukin10 (IL10), transforming growth factor-beta (TGF $\beta$ ) was measured by ELISA in all groups. The data represent the mean \pm SEM of three replications. (B) the transcriptional levels of interleukin10 (IL10), transforming growth factor-beta (TGF $\beta$ ) were analyzed by RTPCR in different groups $\left(\mathrm{n}=3\right.$, mean $\pm \mathrm{SEM},{ }^{*} \mathrm{P}<0.05$ compared to MCAO group). GLGZD, Gualou Guizhi Decoction; MCAO, middle cerebral artery occlusion.
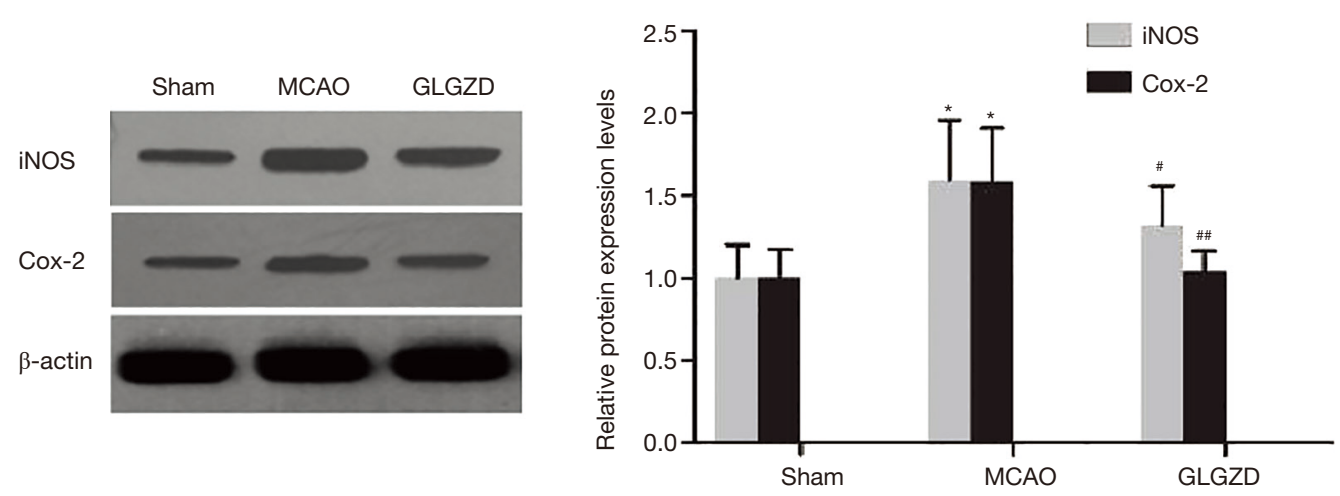

Figure 4 Effect of GLGZD on inducible nitric oxide synthase (iNOS) and cyclooxygenase-2 (Cox-2) expression in MCAO rats. The expression levels of inducible nitric oxide synthase (iNOS) and cyclooxygenase-2 (Cox-2) were detected by Western blotting assay. The experiment was replicated three times. Relative expression levels were determined by densitometry and normalized to actin levels ( $\mathrm{n}=3$, mean \pm SEM, ${ }^{*} \mathrm{P}<0.05$ compared to sham group, ${ }^{\#} \mathrm{P}<0.05,{ }^{\# \#} \mathrm{P}<0.01$ compared to MCAO group). GLGZD, Gualou Guizhi Decoction; MCAO, middle cerebral artery occlusion. 
A
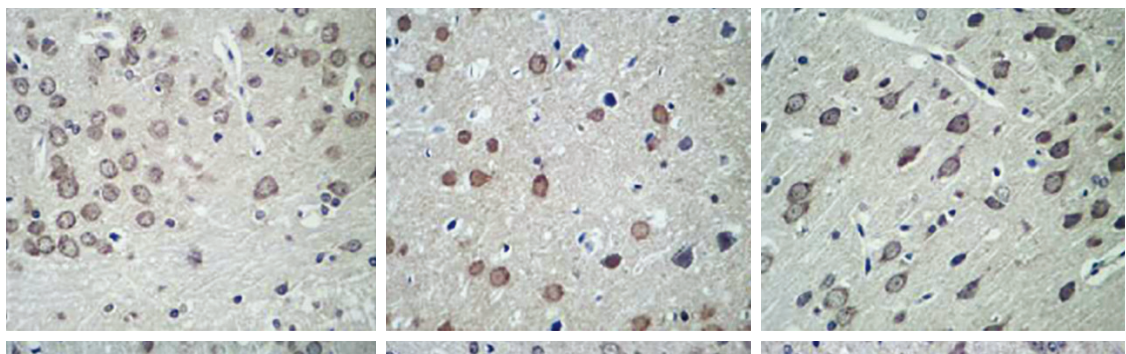

socs1

IKK
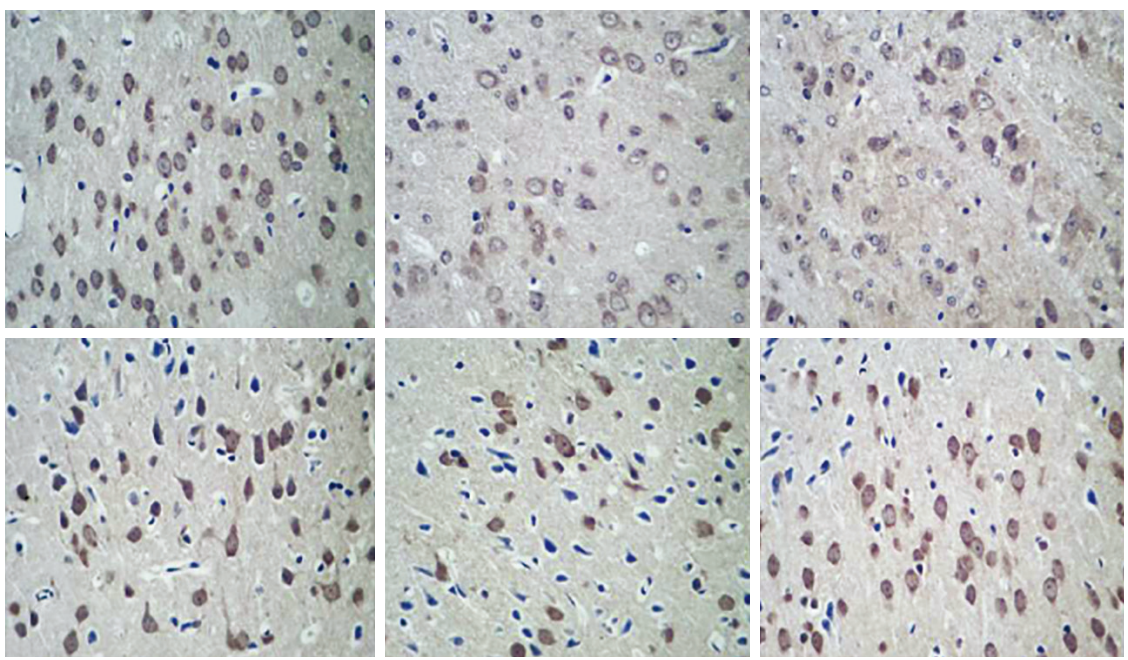

SMAD2
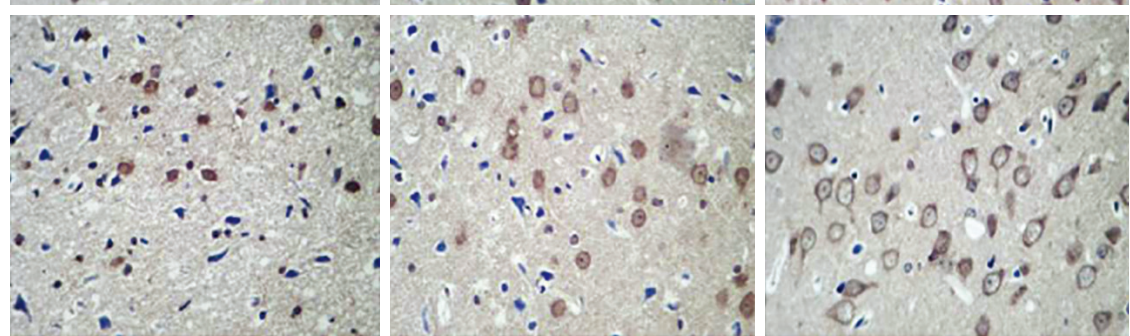

B

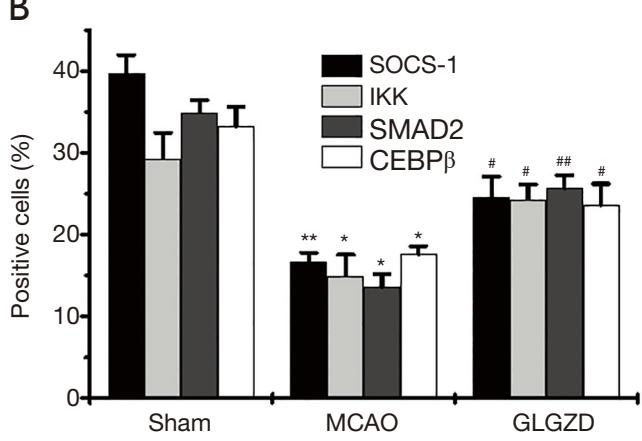

C

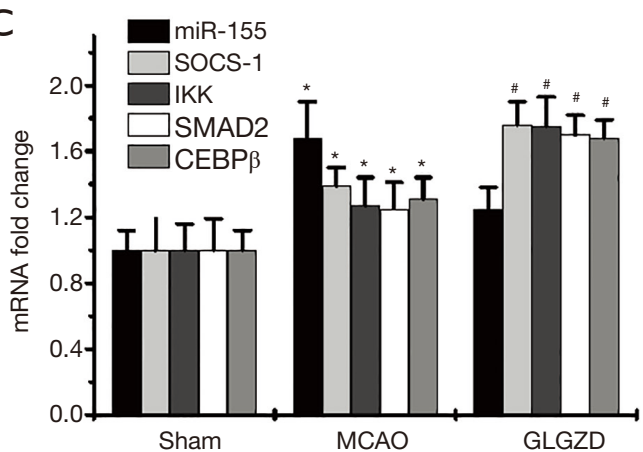

Figure 5 Effect of GLGZD on the expression levels of miR-155 target molecules in MCAO rats. The expression levels of suppressor of cytokine signaling 1 (SOCS1), inhibitor of nuclear factor kappa-B kinase (IKK), mothers against decapentaplegic homolog 2 (SMAD2) and CCAAT/enhancer binding protein beta (CEBP $\beta$ ) were assessed by Immunohistochemistry and RT-PCR assay, respectively. (A) Immunohistochemistry staining for miR155 target molecules in experimental group (400×). (B) Quantification of protein positive cells in the cortex. Data are expressed as the mean $\pm \mathrm{SEM},{ }^{*} \mathrm{P}<0.05$, ${ }^{*} \mathrm{P}<0.01$ vs. sham group; ${ }^{\#} \mathrm{P}<0.05,{ }^{\# \#} \mathrm{P}<0.01$ vs. MCAO group. (C) Semiquantification of miR-155 target molecules in each group as fold increase relative to internal reference. Data are expressed as the mean \pm SEM, ${ }^{*} \mathrm{P}<0.05$ vs. sham group; ${ }^{*} \mathrm{P}<0.05$ vs. MCAO group. GLGZD, Gualou Guizhi Decoction; MCAO, middle cerebral artery occlusion. 
$\mathrm{P}<0.01$ for SMAD2). Conversely, GLGZD treatment dramatically downregulated $\mathrm{miR}-155$ levels and upregulated SOCS1, SMAD2, IKK and CEBP $\beta$ levels as qualified by RT-qPCR and immunohistochemistry (Figure $5 B$ ) in the ischemic ipsilateral brain $(\mathrm{P}<0.05$ compared with the MCAO group).

\section{Discussion}

Ischemic stroke can be divided into permanent or transient blood blockage, either of which can exacerbate stroke severity. There are ample studies confirming the key role of excessive inflammatory response in the central nervous system (CNS) in cerebral ischemia. The post-ischemic neuroinflammatory cascade releases a range of uncontrolled inflammatory cytokines which have the potential to exacerbate neural damage. The balance of pro-inflammatory (IL6, TNF $\alpha$ ) and anti-inflammatory (IL10, TGF $\beta$ ) mediators influence the progression of tissue injury. Recent findings (17) confirmed that noncoding RNAs, especially microRNAs (miRNAs) are involved in I/R injury and play an important role in the progression of the inflammatory response. miRNAs, which are highly conserved small noncoding RNAs, play a crucial role in the modulation of genes that characterize the inflammatory reaction. Several miRNAs have emerged as essential gene expression regulators of inflammatory reaction. Among them, miR-155 is a specific miRNA emerging as a potential biomarker for the pathophysiology of inflammation in neurodegenerative diseases. Incidentally, these studies suggest that miR-155 may double as a potential therapeutic target (18). Previous studies have demonstrated that SOCS1, IKK, SMAD2 and CEBP $\beta$ behaved as downstream target molecules of miR155 during microglial activation $(19,20)$. Additionally, SOCS1 and SMAD2 appeared to negatively regulate the inflammatory response.

Accumulating evidence has revealed that inflammation was closely associated with brain tissue damage. Moreover, the associations between miR-155 and its role in the inflammatory injury of MCAO rats has been confirmed by previous reports. GLGZD is a Chinese herbal compound widely used for the treatment of post-stroke spasticity, displaying strong neuroprotective effects in cerebral ischemic injury. In our previous studies, we found that GLGZD had the potential to improve limb spasms due to inhibitory tissue injury $(11,15)$. Additionally, it has been shown that GLGZD could suppress the inflammatory response through the regulation of the robust release of proinflammatory cytokines. Therefore, the current study set out to investigate the underlying mechanism of GLGZD which modulate ischemic stroke outcomes by regulating the post ischemic inflammatory responses (21-23). Initially, we established the middle cerebral artery occlusion (MCAO) model to explore the mechanism of the protective effects of GLGZD treatment. Consistent with previous studies, the Catwalk gait results in this study revealed that GLGZD could dramatically improve the neurological and functional deficits, including mobility. Furthermore, GLGZD significantly downregulated the expression levels of miR-155, inhibiting enhanced expression observed in MCAO model rats. To further investigate the accurate role of miR-155, we sought to observe the transcriptional and translational levels of miR-155 targets like SOCS1, SMAD2, IKK and CEBP $\beta$, all of which are highly associated with the neuroinflammatory status by IHC and RTPCR. The data in this study showed that GLGZD could effectively upregulate the expression of SOCS1, SMAD2, IKK and CEBP $\beta$ compared to the MCAO group. SOCS1 and SMAD2 were identified as negative regulators of proinflammatory cytokines. Notably, though changes were not observed following ischemia caused by MCAO, GLGZD exerted a promotive effect on the protein and mRNA expression levels of anti-inflammatory cytokines (IL10 and TGF $\beta$ ) released following ischemia, when compared with those of MCAO rats. This result is consistent with previous studies showing novel evidence supporting the notion that GLGZD lead to dramatic decreases in the expression of miR-155 and the marked increase of SOCS1, SMAD2, IKK and CEBP $\beta$ and production of IL10 and TGF $\beta$. These results suggested that GLGZD is a potent agent against the inflammatory responses that are detrimental during ischemic stroke, via miR-155 mediated signaling. The results of this study can be applied to other similar animal models (such as mice), and has great value in the treatment of clinical patients. However, this study was limited to the animal study, furthermore, the outcome of an observational study may be biased by different animals.

In conclusion, these findings provide novel insight for the mechanism of the neuroprotective GLGZD treatment for spasticity following ischemia. It is revealed that GLGZD exerts an inhibitory effect on neuroinflammatory signals contributing to aggravated neuron damage. GLGZD enhances anti-inflammatory factors by reducing miR-155 expression and targeting SOCS1, IKK, SMAD2 and CEBP $\beta$ in the ischemic brain. This study provides support for the utility of GLGZD as a promising therapeutic strategy to 
reduce brain injury closely related to neuroinflammation in ischemic stroke. Further studies should explore other potential mechanisms by which GLGZD may regulate inflammation.

\section{Acknowledgments}

Funding: This study was supported by Open research platform of Fujian University of traditional Chinese Medicine [Laboratory of traditional Chinese medicine pharmacognosy (Level 3), X2019014-platform], Fujian Province Natural Science Foundation(2018J01886) and Fujian Province Natural Science Foundation (2017J01849).

\section{Footnote}

Reporting Checklist: The authors have completed the ARRIVE reporting checklist. Available at http://dx.doi. org/10.21037/apm-20-518

Data Sharing Statement: Available at http://dx.doi. org/10.21037/apm-20-518

Conflicts of Interest: All authors have completed the ICMJE uniform disclosure form (available at http://dx.doi. org/10.21037/apm-20-518). The authors have no conflicts of interest to declare.

Ethical Statement: The authors are accountable for all aspects of the work in ensuring that questions related to the accuracy or integrity of any part of the work are appropriately investigated and resolved. Experiments were performed under a project license (No.: 20190016) granted by the Institutional Animal Care and Use Committee of Fujian University of Traditional Chinese Medicine. Experimental procedures and the care of animals were performed strictly in conformity with international ethical guidelines requirement and the National Institutes of Health Guide concerning the Care and Use of Laboratory Animals.

Open Access Statement: This is an Open Access article distributed in accordance with the Creative Commons Attribution-NonCommercial-NoDerivs 4.0 International License (CC BY-NC-ND 4.0), which permits the noncommercial replication and distribution of the article with the strict proviso that no changes or edits are made and the original work is properly cited (including links to both the formal publication through the relevant DOI and the license). See: https://creativecommons.org/licenses/by-nc-nd/4.0/.

\section{References}

1. Esenwa C, Gutierrez J. Secondary stroke prevention: challenges and solutions. Vasc Health Risk Manag 2015;11:437-50.

2. Brainin M, Norrving B, Sunnerhagen KS, et al. Poststroke chronic disease management: towards improved identification and interventions for poststroke spasticityrelated complications. Int J Stroke 2011;6:42-6.

3. Bethoux F. Spasticity Management After Stroke. Phys Med Rehabil Clin N Am 2015;26:625-39.

4. Wang P, Zhang J, Guo F, et al. Lipopolysaccharide worsens the prognosis of experimental cerebral ischemia via interferon gamma-induced protein 10 recruit in the acute stage. BMC Neurosci 2019;20:64.

5. Shan Y, Tan S, Lin Y, et al. The glucagon-like peptide-1 receptor agonist reduces inflammation and blood-brain barrier breakdown in an astrocyte-dependent manner in experimental stroke. J Neuroinflammation 2019;16:242.

6. Anrather J, Iadecola C. Inflammation and Stroke: An Overview. Neurotherapeutics 2016;13:661-70.

7. Stamatovic SM, Phillips CM, Martinez-Revollar G, et al. Involvement of Epigenetic Mechanisms and Noncoding RNAs in Blood-Brain Barrier and Neurovascular Unit Injury and Recovery After Stroke. Front Neurosci 2019;13:864.

8. Yin KJ, Hamblin M, Chen YE. Non-coding RNAs in cerebral endothelial pathophysiology: emerging roles in stroke. Neurochem Int 2014;77:9-16.

9. Alexandrov P, Zhai Y, Li W, et al. Lipopolysaccharidestimulated, NF-kB-, miRNA-146a- and miRNA-155mediated molecular-genetic communication between the human gastrointestinal tract microbiome and the brain. Folia Neuropathologica 2019;57:211-9.

10. Gaudet AD, Fonken LK, Watkins LR, et al. MicroRNAs: Roles in Regulating Neuroinflammation. Neuroscientist 2018;24:221-45.

11. Han J, Zhang JZ, Zhong ZF, et al. Gualou Guizhi decoction promotes neurological functional recovery and neurogenesis following focal cerebral ischemia/ reperfusion. Neural Regen Res 2018;13:1408-16.

12. Hu J, Pang WS, Han J, et al. Gualou Guizhi decoction reverses brain damage with cerebral ischemic stroke, multi-component directed multi-target to screen calciumoverload inhibitors using combination of molecular 
docking and protein-protein docking. J Enzyme Inhib Med Chem 2018;33:115-25.

13. Nan L, Yang L, Zheng Y, et al. Effects of Gualou Guizhi Decoction Aqueous Extract on Axonal Regeneration in Organotypic Cortical Slice Culture after Oxygen-Glucose Deprivation. Evid Based Complement Alternat Med 2017;2017:5170538.

14. Hu H, Zhu X, Lin R, et al. Suppressive effects of Gua Lou Gui Zhi decoction on MCAO-induced NO and PGE2 production are dependent on the MAPK and NF- $\kappa \mathrm{B}$ signaling pathways. Mol Med Rep 2016;14:5141-7.

15. Huang J, Tao J, Xue X, et al. Gua Lou Gui Zhi decoction exerts neuroprotective effects on post-stroke spasticity via the modulation of glutamate levels and AMPA receptor expression. Int J Mol Med 2013;31:841-8.

16. Longa EZ, Weinstein PR, Carlson S, et al. Reversible middle cerebral artery occlusion without craniectomy in rats. Stroke 1989;20:84-91.

17. Marques-Rocha JL, Samblas M, Milagro FI, et al. Noncoding RNAs, cytokines, and inflammation-related diseases. FASEB J 2015;29:3595-611.

18. Teng G, Papavasiliou FN. Shhh! Silencing by

Cite this article as: Yang $\mathrm{J}$, Zhu $\mathrm{X}, \mathrm{Hu} \mathrm{H}$, Lin $\mathrm{X}$. The inhibitory effect of Gualou Guizhi Decoction on post-ischemic neuroinflammation via miR-155 in MCAO rats. Ann Palliat Med 2021;10(2):1370-1379. doi: 10.21037/apm-20-518
microRNA-155. Philosophical transactions of the Royal Society of London Series B. Biol Sci 2009;364:631-7.

19. Carvalho AF, Berk M, Hyphantis TN, et al. The integrative management of treatment-resistant depression: a comprehensive review and perspectives. Psychother Psychosom 2014;83:70-88.

20. Zheng X, Huang H, Liu J, et al. Propofol Attenuates Inflammatory Response in LPS-Activated Microglia by Regulating the miR-155/SOCS1 Pathway. Inflammation 2018;41:11-9.

21. Zhang Y, Zhang S, Li H, et al. Ameliorative effects of Gualou Guizhi decoction on inflammation in focal cerebral ischemic-reperfusion injury. Mol Med Rep 2015;12:988-94.

22. Hu H, Li Z, Zhu X, et al. Gua Lou Gui Zhi decoction suppresses LPS-induced activation of the TLR4/NF- $\kappa$ B pathway in BV-2 murine microglial cells. Int J Mol Med 2013;31:1327-32.

23. Hu H, Li Z, Zhu X, et al. GuaLou GuiZhi decoction inhibits LPS-induced microglial cell motility through the MAPK signaling pathway. Int J Mol Med 2013;32:1281-6. 